\title{
EFFECT OF PARTICLE DIAMETER ON EXCLUSION-ZONE SIZE
}

\author{
D.T. NHAN \& G.H. POLLACK \\ Department of Bioengineering, University of Washington, Seattle, WA 98195, USA.
}

\begin{abstract}
Particles and solutes are excluded from the vicinity of hydrophilic surfaces, leaving large microsphere-free regions known as exclusion zones (EZs). Prior work had indicated that EZs could extend to distances of up to several hundred micrometers from the nucleating surface. These observations were made on large, extended surfaces, leaving open the question whether EZ size might depend on the characteristic dimension of the excluding surface. We placed one or few ion-exchange-resin beads whose diameters varied from $15 \mu \mathrm{m}$ to $300 \mu \mathrm{m}$ in cuvettes. The beads were suffused with aqueous microsphere suspensions for observing the surfaces' exclusionary behavior. Results showed a direct relation between bead size and EZ size over the full range of bead diameter, implying a similar relation for smaller particles or molecules, perhaps extending beyond the resolution of the light microscope.

Keywords: colloidal particles, exclusion zone, hydrophilic surfaces, ion-exchange resin beads.
\end{abstract}

\section{INTRODUCTION}

Solutes and particles in aqueous suspensions are excluded from the vicinity of hydrophilic surfaces [1].This vicinal region has been termed as the exclusion zone (EZ). EZs persist around surfaces for extended periods - even days following exposure to colloidal suspensions. They are unexpectedly large, commonly in the order of hundreds of micrometers wide. For the past several years our laboratory has been studying EZ character using various flat, cylindrical, and spherical surfaces including ion-exchange resin beads. All surfaces generate EZs. The spherical specimens generally yield large, robust EZs [2,3], but those studied so far have been limited to large radii of curvature. No studies have been carried out on beads of small diameter. The goal of the experiments was to determine EZ size for smaller beads, and particularly whether a functional relation might exist between bead size and EZ size. If so, then extrapolating to levels below those directly observable in the optical microscope might be possible.

\section{EXPERIMENTAL METHODS}

A rectangular chamber was built from a polystyrene block, 4-cm long, 1-cm wide, and 0.3-cm high. A hole $1 \mathrm{~cm}$ in diameter was drilled vertically through the center of the block, and a microscope coverslip was glued to the bottom to complete the chamber. The ion-exchange beads used in these experiments came in three size ranges: 300-600 $\mu \mathrm{m}$ in hydroxide form (Bio-Rad \# 143-7425), 45-100 $\mu \mathrm{m}$ in hydroxide form (Bio-Rad \#143-7425), and 80-250 $\mu \mathrm{m}$ in chloride form (\#140-1341, Bio-Rad). The microspheres used for determining EZ size were surfactant-free sulfate microspheres $1 \mu \mathrm{m}$ in diameter (Invitrogen \#2090-1). They are synthesized with sulfate groups chemically bound to their surfaces, which dissociate in water to give a single negative charge per group. The microspheres were suspended in distilled, deionized water obtained from a Barnstead D3750 Nanopure Diamond purification system (type 1 HPLC grade $(18.2 \mathrm{M} \Omega)$ ). The volume ratio of microsphere to water was 1:500.

For most experiments, one bead was placed on the chamber floor and the aqueous suspension was then poured over the bead. A cover slip was then set on to the top of the chamber. Immediately afterward, the chamber was placed on the stage of a Zeiss Axiovert-35 inverted optical microscope. 
The bead was observed in the bright field mode with a $2.5 \times, 5 \times$, or $10 \times$ objective lens depending on bead size. For optimal clarity and visibility, the hydroxide beads with diameters ranging from 150 to $300 \mu \mathrm{m}$ were viewed under the $2.5 \times$ objective lens, while the hydroxide beads with diameters $45-100 \mu \mathrm{m}$ and chloride beads with diameters $80-250 \mu \mathrm{m}$ were imaged under the $5 \times$ and 10× objective lens.

\section{RESULTS}

Figures 1 and 2 show representative images obtained with chloride and hydroxide beads, respectively. Both types of bead produced clear EZs that were devoid of microspheres. These zones formed uniformly around the beads, regardless of size or type. After some time, however, various swirls and tails developed around the hydroxide beads (Fig. 2).

Figure 3 shows the relation between bead radius and EZ size. EZs were measured at consistent $45^{\circ}$ intervals around the bead, and taken as the span from the bead surface to the far edge of the EZ, or if there were multiple EZs then to the first EZ layer. Measurements were made after exposing the

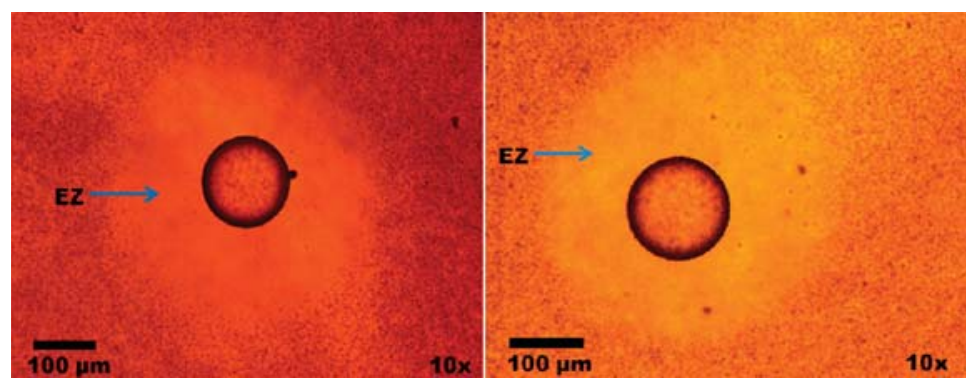

Figure 1: Exclusion zones around chloride beads of different size. Objective magnification is shown on lower right. EZ denotes the microsphere exclusion zone.
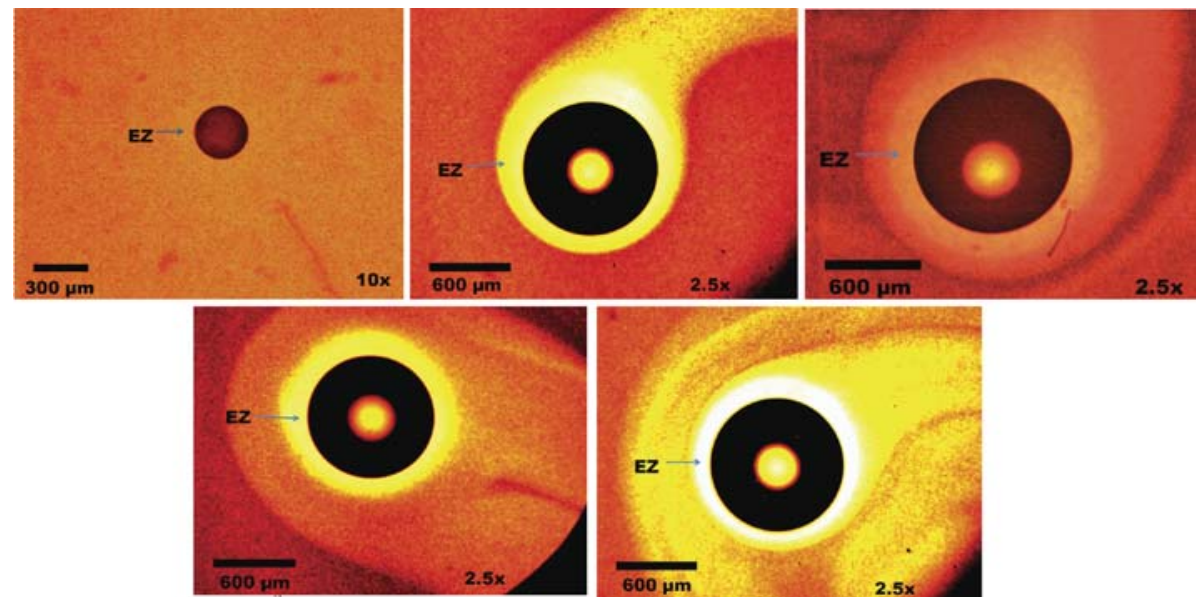

Figure 2: Exclusion zones around hydroxide beads of different size. Swirls and tails shown around beads described further below. Color differences are artifacts stemming from saturation of video camera. 


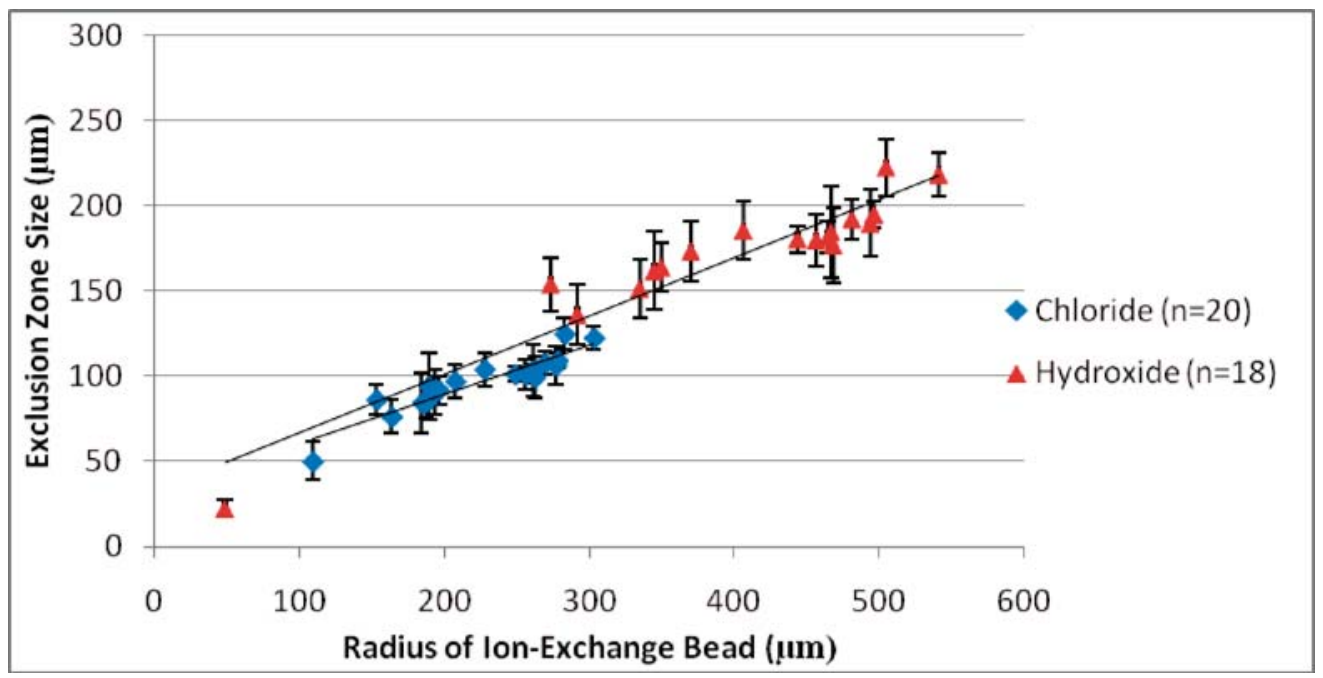

Figure 3: Exclusion zone size as a function of radius for both types of bead.

bead to the microsphere suspension for $25 \mathrm{~min}$. As the hydroxide-bead radius increased from $50 \mu \mathrm{m}$ to $550 \mu \mathrm{m}$, EZ size increased linearly from $120 \mu \mathrm{m}$ to $230 \mu \mathrm{m}$. For the chloride beads, as bead radius increased from $110 \mu \mathrm{m}$ to $330 \mu \mathrm{m}$, EZ size increased linearly from $50 \mu \mathrm{m}$ to $130 \mu \mathrm{m}$.

\subsection{Multiple exclusion-zone layers}

In the large, 300-600 $\mu \mathrm{m}$, hydroxide beads multiple EZs were observed (Fig. 4). After about 5 min, the first EZ layer became stable in width and a second one began to form. At about 15 min that second zone was nearly complete, and a third one began to nucleate. Microspheres tended to complete an existing boundary and fill in any empty pockets before a new EZ began to build. The most common construction was two EZs, which appeared in approximately $70 \%$ of trials, whereas three EZs were noted in some $25 \%$ of trials, and four in approximately $5 \%$. A dense concentration of microspheres marked the distal boundary of each EZ. These multiple layers may have formed as a result of the numerous positive charges anticipated beyond each EZ layer, which should cross-link the negatively charged microspheres and create gel-like entities that could then nucleate an additional EZ.

\subsection{Tails}

Long tail-like EZ structures were another common feature of hydroxide beads, appearing in about $75 \%$ of trials. Generally, the tails extended from the first EZ outward toward the edge of the chamber. After completion of the first EZ, the tail would extend in a single direction and microspheres along the edge of the tail would flow in a direction parallel to the direction of tail growth. Tail structures were sometimes straight, but sometimes also curved in a variety of spiral patterns.

\subsection{Swirls}

Even without microscopy, swirled structures emanating from the hydroxide beads were visible with the naked eye. The swirls began forming at about the 15-min mark and were prevalent in about $20 \%$ 

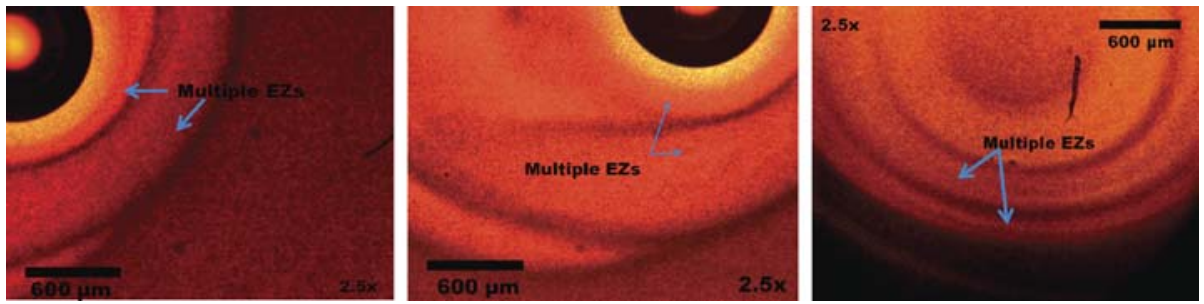

Figure 4: Multiple Exclusion zones (EZs) around hydroxide ion-exchange beads recorded at 25 min. Some of the EZs are not completely microsphere free, but contain microspheres in considerably lower concentration than flanking regions.

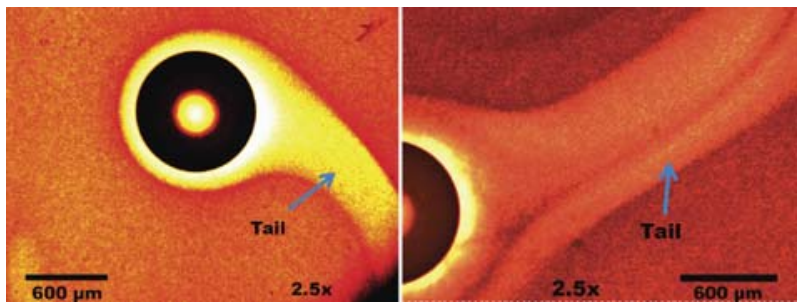

Figure 5: Long, stream-like tails around hydroxide beads, recorded at the 15-min mark.

of trials. They began growing after the first EZ was completed and a tail (Fig. 5) began to emanate. At about the 25-min mark, about half of the trials that formed swirls showed a single rotation while the rest showed multiple rotations. Swirl dimensions were measured from origin to tip and ranged from $400 \mu \mathrm{m}$ to as large as $2,000 \mu \mathrm{m}$. These sizes had no apparent correlation with bead diameter. Swirls were only found with the hydroxide beads and not with the chloride beads.

\section{DISCUSSION}

The main finding of these experiments was that EZ size depends on the shape of the nucleating surface. We found that the smaller the bead, the smaller the EZ. This was seen whether the bead was of the hydroxide type or the chloride type (Fig. 3).

Surface curvature evidently impacts EZ size, and the question is why this is so. The EZ is a relatively more ordered zone of water adjacent to some nucleating surface. Envisioning growth of such an ordered zone adjacent to a flat surface is straightforward. However, convex surfaces imply raylike divergence, and a possibility is that any such divergence has negative impact on size because the growing domains disconnect. That would explain why smaller curvature radii gave smaller EZ size. And it would also explain why flat surfaces produce EZs larger than those found here.

A question that arises is the EZ size value adjacent to smaller particles including those not easily measurable by ordinary optical microscopy. The EZ is a feature common to many if not all hydrophilic surfaces [4]. If so, then they would also occur adjacent to very small particles and molecules. Generally the water-containing zones adjacent to such surfaces are considered as hydration and presumed to extend no more than several molecular layers from the surface. But the extensive EZs noted here and elsewhere imply that such 'hydration' layers could be larger and perhaps much larger. 

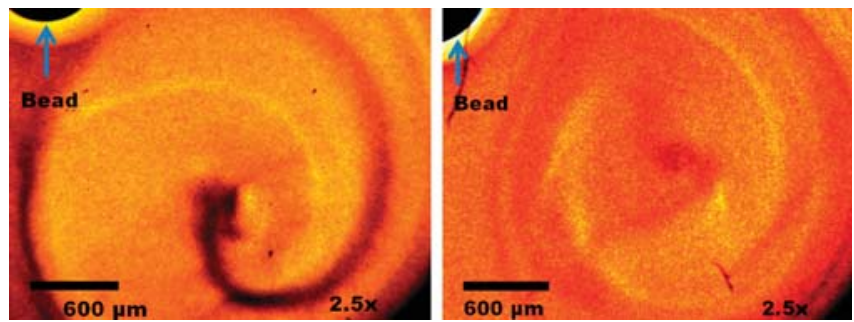

Figure 6: Swirls formed from a hydroxide bead at $25 \mathrm{~min}$.

The results obtained here cannot answer the question of what happens with small curvatures but they provide a clue. If the trend follows, then smaller scale nucleators should produce correspondingly small EZs. Extrapolating the graph of Fig. 3 to zero radius gives a small but non-zero EZ size, although the zone of extrapolation would seem too large to be reliable.

On the other hand, indirect evidence implies that the extrapolation may be valid down at least to the micron scale. The data of Fig. 3 show EZ values roughly half of the respective bead radius. For a $1-\mu \mathrm{m}$ sphere, that would imply an EZ on the order of $0.5 \mu \mathrm{m}$. Experiments on Brownian dynamics [5] demonstrate an EZ on the micron to sub-micron scale, hence within the range expected. Thus, the extrapolation of Fig. 3 would seem to hold at least down to the micrometer scale. Whether it holds down to the molecular scale remains to be determined.

Notable features of the results are the multiple EZs, tails and swirls (Figs 4-6). These features were found with the hydroxide beads but not the chloride beads, implying that liberated protons might play a role [6]. The multiple EZs might arise from proton abundance: if protons link microspheres by the like-likes-like mechanism [7], then the linked microspheres form a gel-like entity, which could itself nucleate another EZ. Hence multiple EZs would appear with time. Such multiple zones have previously been seen adjacent to flat surfaces; hence they are not necessarily features associated with curvature. They are quite common.

The tails may arise as extensions of the EZ. Such extensions arise commonly in EZs adjacent to flat Nafion surfaces. The ordinary EZ typically reaches a terminal width, but continue their growth with time by projecting narrow extensions normal to the plane of the EZ. Such zones can project up to $1 \mathrm{~m}$ or farther [8]. When even low-level flow is present parallel to the nucleating surface these extensions will deflect, yielding the swirls that were commonly seen in the hydroxide beads. Again, if the protons, or proton gradients, are responsible for any such flows, then this might explain why the swirls appear much more routinely with the hydroxide beads than with the chloride beads.

In conclusion, the results have shown that surfaces with smaller radii of curvature generate smaller EZs. EZs are by no means constant in size. They depend on various factors, not the least of which is the characteristic dimension of the nucleating surface.

\section{REFERENCES}

[1] Zheng, J.M. \& Pollack, G.H., Long range forces extending from polymer surfaces. Phys Rev E, 68, p. 031408, 2003. doi:10.1103/PhysRevE.68.031408

[2] Nagornyak, E., Yoo, H. \& Pollack, G.H., Mechanism of attraction between like-charged particles in aqueous solution. Soft Matter, 5, pp. 3850-3857, 2009. doi:10.1039/b905080a

[3] Zheng, J.M., Wexler, A. \& Pollack, G.H., Effect of buffers on aqueous solute-exclusion zones around ion-exchange resins. J. Colloid Interface Sci., 332, pp. 511-514, 2009. doi:10.1016/j.jcis.2009.01.010 
[4] Zheng, J.M. \& Pollack, G.H., Solute exclusion and potential distribution near hydrophilic surfaces. Water and the Cell, ed. G.H. Pollack, I.L. Cameron, \& D.N. Wheatley, Springer, The Netherlands, pp. 165-174, 2006.

[5] Bhalerao, A. \& Pollack, G.H., Light-induced effects on Brownian displacements, submitted. J Biophotonics, 1-7, 2010, doi: 10.1002/jbio.201000097.

[6] Chai, B., Yoo, H. \& Pollack, G.H., Effect of radiant energy on near-surface water. J. Phys. Chem. B, 113, pp. 13953-13958, 2009.

[7] Zhao, Q., Zheng, J.M., Chai, B. \& Pollack, G.H., Unexpected effect of light on colloid crystal Spacing. Langmuir, 24, pp. 1750-1755, 2008.

[8] Yoo, H., Baker, D.R., Pirie, C.M., Hovakeemian, B. \& Pollack, G.H., Characteristics of water adjacent to hydrophilic interfaces. Water, the Forgotten Biological Molecule, ed. D. LeBihan \& H. Fukuyama, Pan Stanford Publishing Pte. Ltd., 2010. 